\title{
Long-Term Rice and Green Manure Rotation Alters the Endophytic Bacterial Communities of the Rice Root
}

\author{
Xiao-Xia Zhang • Ju-Sheng Gao • Yan-Hua Cao • \\ Xiao-Tong Ma $\cdot$ Ji-Zheng He
}

Received: 16 June 2013 / Accepted: 5 September 2013 / Published online: 18 September 2013

(C) Springer Science+Business Media New York 2013

\begin{abstract}
This study focuses on the effects of long-term rice rotated with milk vetch being as green manure on the composition of bacteria in rice roots. The endophytic bacterial communities in rice roots of the rice-rice-milk vetch (R-R-MV) and the rice-rice-winter fallow (R-R-WF) crop rotations with a 28-year research history were investigated using combined culture-dependent and culture-independent methods. It was found that the endophytic bacterial population in rice roots with the green manure was significantly higher than that of without it. There were 169 and 77 strains of endophytic bacteria that were isolated from rice roots of the R-R-MV and the R-R-WF, respectively. The 16S rRNA gene analysis shows that the 77 R-R-WF bacteria belong to 15 species of 14 genera while the other 169 R-R-MV bacteria belong to 21 species of 19 genera, in which Herbaspirillum and Cedecea were two mutually dominant populations and Burkholderia, Pseudomonas, Sphingomonas, and Pantoea accounted for large proportions of the endophytic bacteria in rice roots
\end{abstract}

Electronic supplementary material The online version of this article (doi:10.1007/s00248-013-0293-1) contains supplementary material, which is available to authorized users.

X.-X. Zhang · J.-S. Gao $\cdot$ Y.-H. Cao $\cdot$ X.-T. Ma

Key Laboratory of Microbial Resources Collection and Preservation, Ministry of Agriculture, Institute of Agricultural Resources and Regional Planning, Chinese Academy of Agricultural Sciences, Beijing 100081, China

\section{J.-Z. He $(\bowtie)$}

State Key Laboratory of Urban and Regional Ecology, Research Center for Eco-Environmental Sciences, Chinese Academy of Sciences, Beijing 100085, China

e-mail: jzhe@rcees.ac.cn

J.-S. Gao

Qiyang Agro-ecosystem of National Field Experimental Station, Institute of Agricultural Resources and Regional Planning, Chinese Academy of Agricultural Sciences, Qiyang 426182, China through R-R-MV rotation. The analysis of $16 \mathrm{~S}$ rDNA clone libraries showed that the Shannon-Weaver diversity index of endophytic bacteria in R-R-MV approximates that in R-R-WF rotation, whereas the richness indexes of Chao 1 and $\mathrm{ACE}$ in R-R-MV rotation system were significantly higher than those in R-R-WF rotation. The diversity of endophytic bacteria was richer in R-R-MV. Both the culture-dependent and the cultureindependent method revealed significant effect of long-term different tillage systems on the microbial community.

\section{Introduction}

Green manure plays a positive role in reducing soil erosion, improving soil physical properties [19], lifting organic matter and fertility levels, extending the nutrient persistence [4], and restraining global warming potentials [25], and it also contributes to higher crop yields $[6,32]$. It is one of the sustainable agricultural measures to maintain and improve soil quality [32]. Soil microbes and their life processes serve as a vital factor influencing the long-term development of agricultural ecosystem [1]. Therefore, an in-depth and systematic research on the effects of green manure on soil microbes is of great importance. Tejada et al. [32] applied green manure over four consecutive years and found that various green manure had significant impact on the yields of corn, soil microbial biomass, dehydrogenase, urease, glucosaccharase, phosphatase, and arylsulfatase. Stark et al. [29] studied the effects of the green manure lupin on soil microbes and enzyme activity. Yang et al. [35] discovered the impact of different kinds of green manure on soil microbial biomass and enzyme activity. Although these existing reports could prove the significant effect of green manure on soil microbes, the research methods on it only made indirect assessment by means of microbial biomass, enzyme activity characteristics, etc. [29, 32]. 
Large amount of researches have demonstrated that endophytic bacteria in plants could promote plant growth through nitrogen fixation, siderophore production, and secretion of plant growth regulating substances such as auxins, cytokinins, and gibberellins, 1-aminocyclopropane-1-carboxylate deaminase [3]. It has great application potentials in agricultural production [22]. The endophytic bacteria in the plant have been generally considered to be from rhizosphere soil, phylloplane, and seeds. They were influenced by the host's living conditions [20]. Long-term research studies on the ricecollecting zone have confirmed that, compared with R-R-WF system, the rice yield of long-term green manure rotation increased $54,081 \mathrm{~kg} / \mathrm{hm}^{2}$, up by $26.4 \%$; the production of rice straw increased $44,109 \mathrm{~kg} / \mathrm{hm}^{2}$, up by $27.1 \%$ [7]. However, there is no information available about the effects of rotation on rice root endophytic bacterial community. We presume that long-term green manure rotation can enhance the richness and diversity of crop endophytic bacteria. This study combined culture-dependent and culture-independent methods to explore the influence of long-term green manure rotation on the diversity of endophytic bacteria and their functional colonies in rice, providing a theoretical basis for the research of effects of long-term green manure rotation on rice production and obtaining endophytic bacterial resources in rice.

\section{Materials and Methods}

\section{Experiment Design and Site Description}

The site for long-term crop rotation experiment is located at the Key Field Monitoring Experimental Station for Red Soil Eco-environment of Ministry of Agriculture, Qiyang County of Hunan Province $\left(26^{\circ} 45^{\prime} 42^{\prime \prime} \mathrm{N}, 111^{\circ} 52^{\prime} 32^{\prime \prime} \mathrm{E}\right)$. This rotation experiment involved two tillage systems: rice-rice-winter fallow (R-R-WF) and rice-rice-milk vetch (R-R-MV). Each treatment was repeated three times. Each plot covered an area of $37.5 \mathrm{~m}^{2}(2.5 \mathrm{~m} \times 15.0 \mathrm{~m})$ and was separated by a $60-\mathrm{cm}$ cement bund. From 1982 to 2010, the total fertilizer (base fertilizer + topdressing) applied to the early and late rice of each season includes $\mathrm{N}\left(153.0 \mathrm{~kg} / \mathrm{hm}^{2}\right), \mathrm{P}_{2} \mathrm{O}_{5}\left(84.0 \mathrm{~kg} / \mathrm{hm}^{2}\right)$, and $\mathrm{K}_{2} \mathrm{O}\left(129.0 \mathrm{~kg} / \mathrm{hm}^{2}\right)$. Compound fertilizer [N $(84.0 \mathrm{~kg} /$ $\left.\mathrm{hm}^{2}\right), \mathrm{P}_{2} \mathrm{O}_{5}\left(84.0 \mathrm{~kg} / \mathrm{hm}^{2}\right)$, and $\left.\mathrm{K}_{2} \mathrm{O}\left(84.0 \mathrm{~kg} / \mathrm{hm}^{2}\right)\right]$ was used as base fertilizer and $\mathrm{N}\left(69.0 \mathrm{~kg} / \mathrm{hm}^{2}\right)$ and $\mathrm{K}_{2} \mathrm{O}\left(45.0 \mathrm{~kg} / \mathrm{hm}^{2}\right)$ were used as topdressing. The base fertilizer was applied before rice transplanting and topdressing was applied 6 to 10 days after rice transplanting. Milk vetch was sown in winter. Ten to 15 days before the harvest of late rice, milk vetch seeds were spread onto the plot with an amount of $37.5 \mathrm{~kg} / \mathrm{hm}^{2}$. Fresh straw from the plots where milk vetch had been grown was returned into the original plots 15 days before early rice transplanting. All straw (except rice stubble) was removed from the plots after each season of rice harvest. No fertilizer was applied to the green manure planted in winter.

\section{Sampling}

The tillering-stage rice roots were sampled from long-term experiments of R-R-MV and R-R-WF tillage systems in September of 2010 and placed on ice. After brought to the lab, they were cleaned to remove the silt from the surface and preserved in refrigerators at 4 and $-70{ }^{\circ} \mathrm{C}$.

\section{Surface Sterilization of Rice Roots}

Ethanol-sodium hypochlorite was used for surface sterilization of rice roots as described by Sun et al. [30] and Garbeva et al. [8]. Soil on the surface of rice roots was washed off with sterile water and then the roots were dipped in $70 \%$ ethanol for $3 \mathrm{~min}$ and in $2.6 \%$ sodium hypochlorite for $5 \mathrm{~min}$ in turn, after which they were washed with $70 \%$ ethanol for $30 \mathrm{~s}$ and then with sterile water for five times. Finally, sterile filter paper was used to absorb extra moisture. The water used for the final wash was spread on the TSA plate so as to examine the surface sterilization effect.

\section{Counts and Isolation of Endophytic Bacteria}

The quantities of endophytic bacteria in rice roots were counted by the dilution plate spread method. A sample $(1 \mathrm{~g})$ after surface sterilization was cut up, fully ground in a sterile mortar with a small amount of sterile quartz sand, and then diluted with sterile water into suspension $10^{-2}, 10^{-3}$, and $10^{-4}$. One hundred microliters was spread on $\mathrm{R}_{2} \mathrm{~A}$ (Difico) medium plate, each treatment with three parallels. Bacteria were incubated at $28^{\circ} \mathrm{C}$ for 3 days. Isolation and count of colonies were made in those plates containing 30 to 300 colonies. All colonies were isolated from one plate under each treatment and all the isolated strains were preserved by freeze-drying method and stored at $4{ }^{\circ} \mathrm{C}$.

ARDRA of Cultivated Endophytic Bacteria and Sequencing of 16S rRNA

Bacterial genome DNA was extracted using kits (TIANGEN) as the template of PCR reaction. Universal primers of $799 \mathrm{~F}$ (5'-AACAGGATTAGATACCCTG-3') [2] and 1492R (5'GGTTACCTTGTTACGACTT-3') [16] were used to amplify fractions of the 16S rDNA gene. PCR products were enzyme-digested with two kinds of restriction endonuclease which are HaeIII and RsaI (TaKaRa) and then went through $4 \mathrm{~h}$ reaction at $37{ }^{\circ} \mathrm{C}$. Afterwards, electrophoresis of $100 \mathrm{~V}$ was made for enzyme-digested products in $2.5 \%$ agarose gel for $60 \mathrm{~min}$. 
Diversity Analysis of Cultivated Endophytic Bacteria

Clustering analysis of the enzyme digestion and typing pattern was made with GelCompar II (Applied maths, Belgium) gel analysis software to determine the operational taxonomic units (OTU) of cultivated strains. Strains were selected according to the fingerprints of enzyme digestion to amplify and sequence the $16 \mathrm{~S}$ rRNA gene. In this study, variance analysis was made with SPSS13.0 and the diversity indexes of cultivated communities, such as Margalef index $\left(d_{\mathrm{Mg}}\right)$, Shannon-Wiener index $\left(H^{\prime}\right)$, and Simpson index $(D)$, were calculated using methods described by Hill et al. [12].

\section{Construction of 16S rDNA Clone Library of Endophytic} Bacteria

Following instructions, whole DNA was extracted and purified from rice roots after surface sterilization using FastDNA ${ }^{\circledR}$ SPIN Kit for Soil. 16S rDNA fractions were amplified with universal primers $799 \mathrm{~F}$ and 1492R. Electrophoresis was conducted for amplification products in $1.7 \%$ agarose gel. About 730 bp DNA fragment was cut off in an ultraviolet light with a sterile blade and then purified (OMEGA). The purified PCR products were ligated into the pEASY-T1 vector according to the protocol supplied by the manufacturer (TRANS Co., China). Escherichia coli DH5 $\alpha$ competent cells (TRANS Co., China) were transformed with the ligation products and spread onto Luria-Bertani agar plates with ampicillin $\left(100 \mu \mathrm{g} \mathrm{ml}^{-1}\right)$ and X-gal/IPTG on the surface for standard blue and white screening. The obtained sequences were checked by Chimera-Check program to remove the artificial chimeric sequences and identified by the EzTaxon-e server (http://eztaxon-e.ezbiocloud.net/) [15].

\section{Phylogenetic Analyses}

A total of 590 sequences of the 16S rRNA gene were analyzed. Sequence alignment was performed using the program ClustalX version 1.83 [33] with default parameters. Pairwise distances between the aligned sequences were performed using the dist.seqs and cluster tools in MOTHUR using default setting. The OTUs were defined at $97 \%$ similarity cutoff for diversity and rarefaction curves were estimated by using MOTHUR (http://www.mothur.org/) [27]. To estimate the representation of the phylotypes, the clone coverage was calculated with the following equation: $C=(1-\mathrm{nl} / N) \times 100 \%$, where $\mathrm{nl}$ is the number of single clones and $N$ is the total number of clones in the clone library [9]. Phylogenetic trees were analyzed by using the software package MEGA version 5.0 [31], applying the neighbor-joining method and distance options according to the Kimura two-parameter model. Neighbor bootstrap values were calculated based on 1,000 replications.
Nucleotide Sequence Accession Numbers

All of the sequences are deposited in GenBank. The series numbers of cultivated bacteria are JX531675-JX531696 and JX446583, and the clone library obtains the GenBank accession no. of KC415620-KC415689.

\section{Results}

Quantification of Bacterial Populations in Rice Roots

Bacterial colonies from roots of the tillering rice using the traditional gradient dilution plate separation methods were counted, revealing that the number in rice roots after R-RWF rotation was $4.9 \times 10^{6} \mathrm{cfu} / \mathrm{g}$ and that after R-R-MV rotation was $9.1 \times 10^{6} \mathrm{cfu} / \mathrm{g}$ on average. Variance analysis revealed significant difference between them $(P<0.005)$. One of the three parallel $\mathrm{R}_{2} \mathrm{~A}$ culture medium plates, which had the most colonies evenly distributed, was selected and all colonies on it were isolated and purified. It obtained 77 isolates from R-R-WF samples and 169 isolates from R-RMV samples.

\section{Diversity Analysis of Cultivated Endophytic Bacteria}

The amplification of the 16S rDNA gene from the 246 cultivated endophytic bacterial isolates was performed using the universal primers $799 \mathrm{f}-1492 \mathrm{r}$. The amplification products were digested using the restriction enzymes HaeIII and RsaI. An OTU was defined as a group of clones that had identical banding patterns obtained from the two independent digestions [30]. Clustering analysis was made on the ARDRA patterns of the 246 cultivated endophytic bacteria using the GelComparII software, from which 23 OTUs were obtained, including 15 OTUs from R-R-WF rice roots and 21 OTUs from R-R-MV rice roots.

According to the ARDRA patterns of the two samples, Margalef index, Shannon-Wiener index, and Simpson index were calculated as shown in Table 1 , indicating that the diversity level of cultivated endophytic bacteria in R-R$\mathrm{MV}$ rice roots was significantly higher than that in R-RWF rice roots.

Table 1 Comparison of OTU numbers, richness, and diversity based on the 16S-ARDRA patterns

\begin{tabular}{lllll}
\hline & No. OTUs & $\begin{array}{l}\text { Margalef } \\
\text { index }\left(d_{\mathrm{Mg}}\right)\end{array}$ & $\begin{array}{l}\text { Shannon-Wiener } \\
\text { index }\left(H^{\prime}\right)\end{array}$ & $\begin{array}{l}\text { Simpson } \\
\text { index }(D)\end{array}$ \\
\hline R-R-WF & 15 & 3.31 & 2.09 & 0.77 \\
R-R-MV & 21 & 3.90 & 2.41 & 0.87 \\
\hline
\end{tabular}


Sequencing of Cultivated Endophytic Bacteria and Phylogenetic Analysis

One to two strains representing each distinct ARDRA pattern was randomly chosen for sequencing. The sequenced fraction of the 16S rRNA gene is around $700 \mathrm{bp}$. The phylogenetic tree of cultivated bacteria was constructed using Mega 5.0 (Fig. 1).

The phylogenetic analysis of the 16S rRNA gene showed that the 77 bacterial isolates from R-R-WF rice roots belonged to 15 species of 14 genera while the 169 bacteria isolated from R-R-MV rice roots belonged to 21 species of 19 genera. In these two samples, most isolates were Proteobacteria, among which, $\beta$-proteobacteria predominates, accounted for 63.6 and $52.7 \%$ of bacteria from R-R-WF and R-R-MV rice roots, respectively. The second largest group was $\gamma$-proteobacteria, accounting for 28.6 and $30.8 \%$, followed by $\alpha$-proteobacteria and Firmicutes. Actinobacteria were only isolated from R-RMV rice roots. In terms of the genus level as presented in Fig. 2, the genus Herbaspirillum was the common dominant population isolated in both treatments (49.6 and $27.2 \%$ in R$\mathrm{R}-\mathrm{WF}$ and R-R-MV rice roots in turn). The second dominant genus in R-R-WF rice roots was Cedecea (20.8 \%) and other genera accounted for $29.9 \%$. Whereas in R-R-MV rice roots, the second dominant groups were Burkholderia (14.8\%), Pantoea (14.2\%), Sphingomonas (10.7\%) Cedecea (8.9\%), and Pseudomonas (5.9\%); others accounted for $18.5 \%$. Pandoraea was specific to R-R-WF rice roots; the genera Haematobacter, Rhizobium, Chromobacterium, Enterobacter, and Microbacterium were specific to R-RMV rice roots. Besides, one strain ZYY136 isolated from R$\mathrm{R}-\mathrm{MV}$ rice roots might stand for a novel bacterial species of the genus Rhizobium.

Statistical and Phylogenetic Analysis of the Clone Library

\section{Coverage (C) Value and Rarefaction Curve}

The number of effective sequences obtained from the R-RWF and R-R-MV treatments was 280 and 310, respectively. The similarity of DNA sequences were compared by using the Mothur and sequences with the similarity of $97 \%$ or higher were grouped into a same OTU. In this way, the 280 sequences from R-R-WF system were grouped into 177 OTUs and the 310 sequences from the long-term R-R-MV system were grouped into 182 OTUs. The size of the clone library was illustrated through calculation of the coverage value $(C)$ and production of the rarefaction curve. The coverage $(C)$ value of the clone library of the $16 \mathrm{~S}$ rDNA gene from the long term R-R-WF system was $22.6 \%$, while the value of the clone library for R-R-MV system was $20.9 \%$. As shown in Fig. 3, the $C$ value was relatively small but the rarefaction curve tended to plateau, indicating the size of the clone library was
Fig. 1 16S rRNA gene-based dendrogram illustrates the phylogenetic relationship of cultivated isolates from the rice roots of rice-rice-winter follow $(R-R-W F)$ and rice-rice- milk vetch $(R-R-M V)$ rotation. Phylogenies are inferred using neighbor-joining analysis and trees are generated using MEGA 5.0 software. Numbers in parentheses represent the sequence accession numbers in GenBank. Numbers at branch points indicate bootstrap values based on 1,000 replicates. The scale bar represents a $2 \%$ estimated difference in nucleotide sequence. The figure after "R-R-WF" and "R-R-MV" stands for the number of the same ARDRA pattern strains

sufficient to represent the microbial diversity of endophytic bacterial communities in rice roots.

\section{Comparative Analysis of Uncultured Endophytic Bacteria Diversity}

The study used the Mothur to count the genetic matrix of the 280 sequences of endophytic bacteria from R-R-WF rotation and the 310 sequences from the long-term R-R-MV rotation, and then classified the OTUs and made diversity statistics using the same software. Diversity counted at evolutionary distance of $97 \%$ (Table 2) showed that the Shannon-Weaver indexes of the endophytic bacteria from R-R-MV and R-RWF rotations were 4.84 and 4.68, respectively, the Simpson index of the former (0.02) was over twice of the index of the latter (0.01), and the Chao 1 and ACE richness indexes of the former were also significantly higher than those of the latter. According to the comparative analysis, the endophytic bacteria diversity level in roots of rice after R-R-MV rotation was significantly higher than that of rice after R-R-WF rotation.

\section{Phylogenetic Comparative Analysis of Uncultured Endophytic Bacteria Communities}

The Mothur software was used to compare the similarity of DNA sequences. Sequence with similarity of $85 \%$ or above are grouped into the same OTU, which means the genetic distance threshold value is 0.15 at the class level. Sequences were calculated with the Mothur software out of the two libraries and a phylogenetic tree generated (supplementary material Fig. S1). Four dominant classes were found out from rice roots with R-R-WF and R-R-MV rotations: Proteobacteria, Firmicutes, Bacteroidetes, and Acidobacteria, which vary enormously in terms of respective proportions. In addition, several other unusual communities are present as well, such as Nitrospirate, Armatimonadetes, Spirochaetes, Chlorobi, Verrucomicrobia, and Ingvibacteriae.

From the class-level comparison as illustrated in Fig. 4, RR-WF and R-R-MV rice shared the Proteobacteria as the dominant colony, which accounted for 68.6 and $74.1 \%$ of the total cloned number under the two treatments, respectively. The Proteobacteria in both samples were consisted of $\alpha$ proteobacteria, $\beta$-proteobacteria, $\gamma$-proteobacteria, and $\delta$ - 


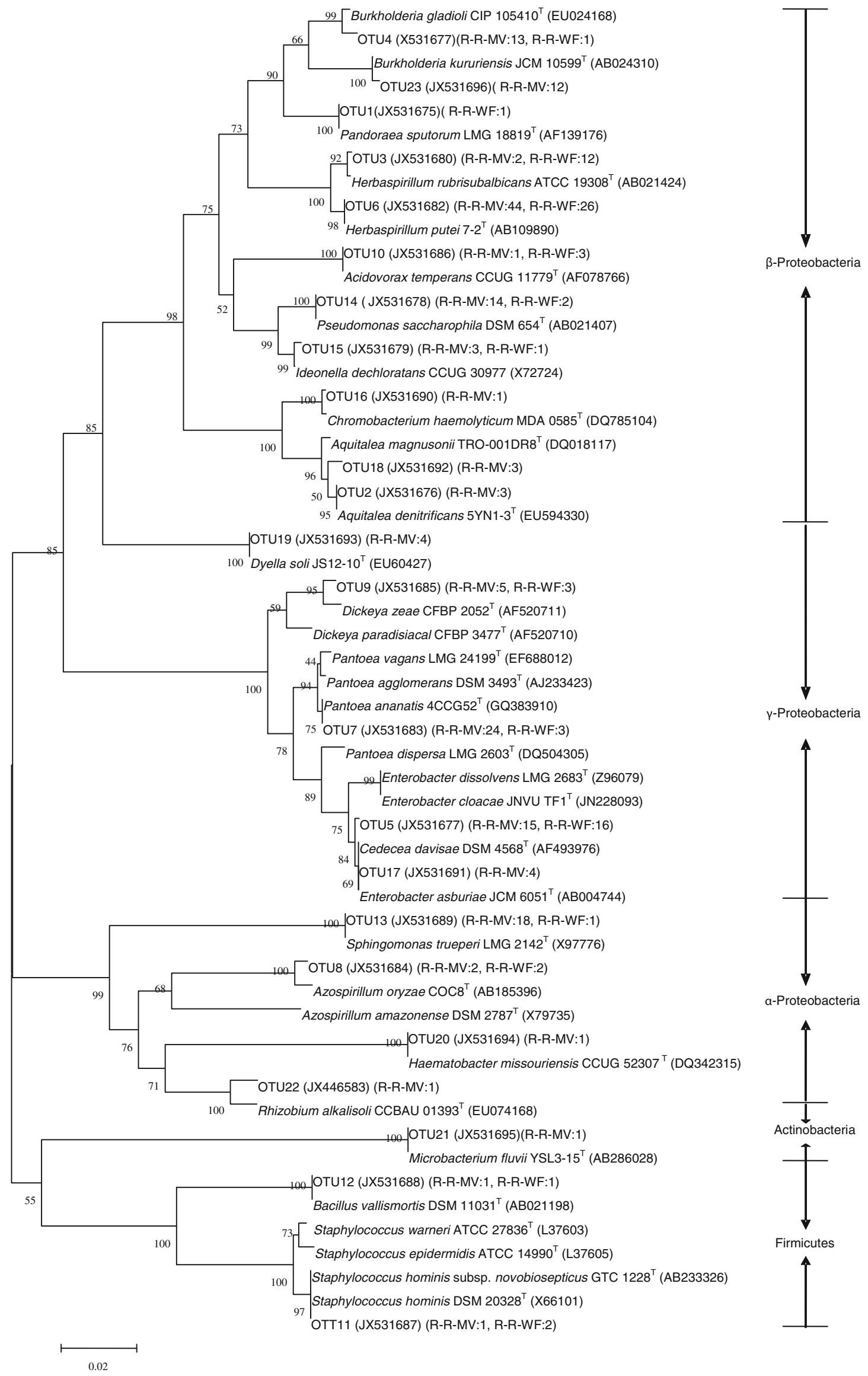


Fig. 2 Genus level comparison of endophytic cultivated bacteria distribution in R-R-WF (a) and R-R-MV (b) rice roots based on culture-dependent method

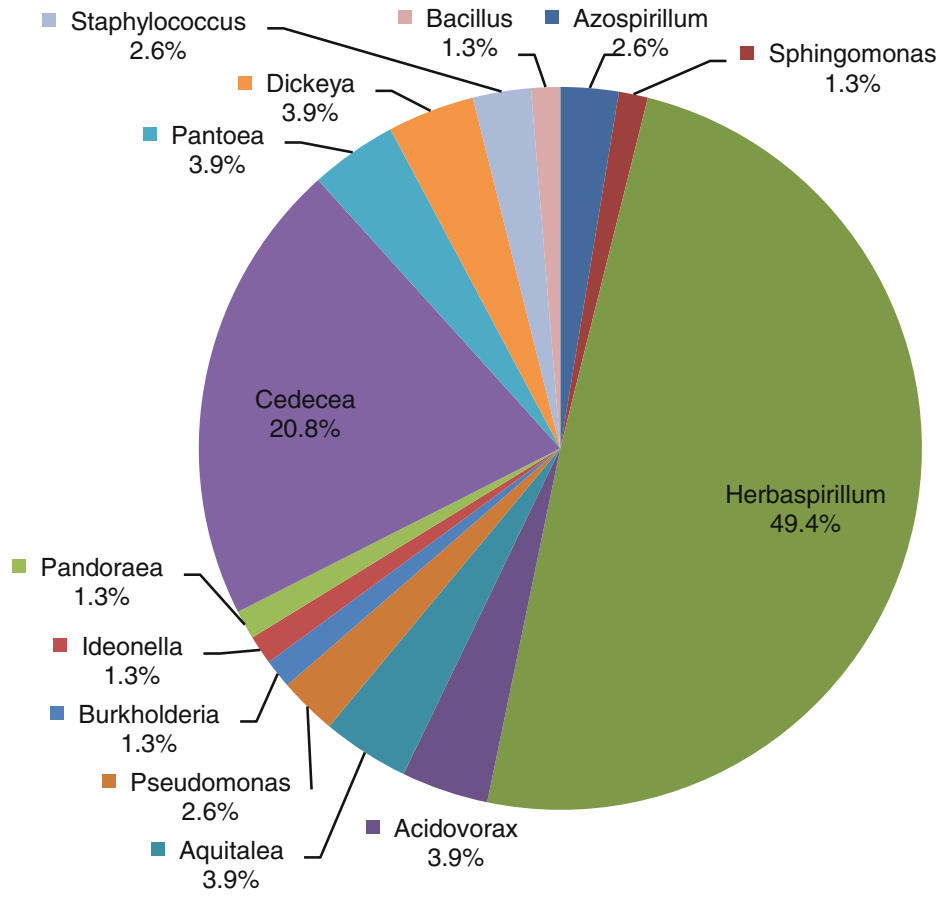

A

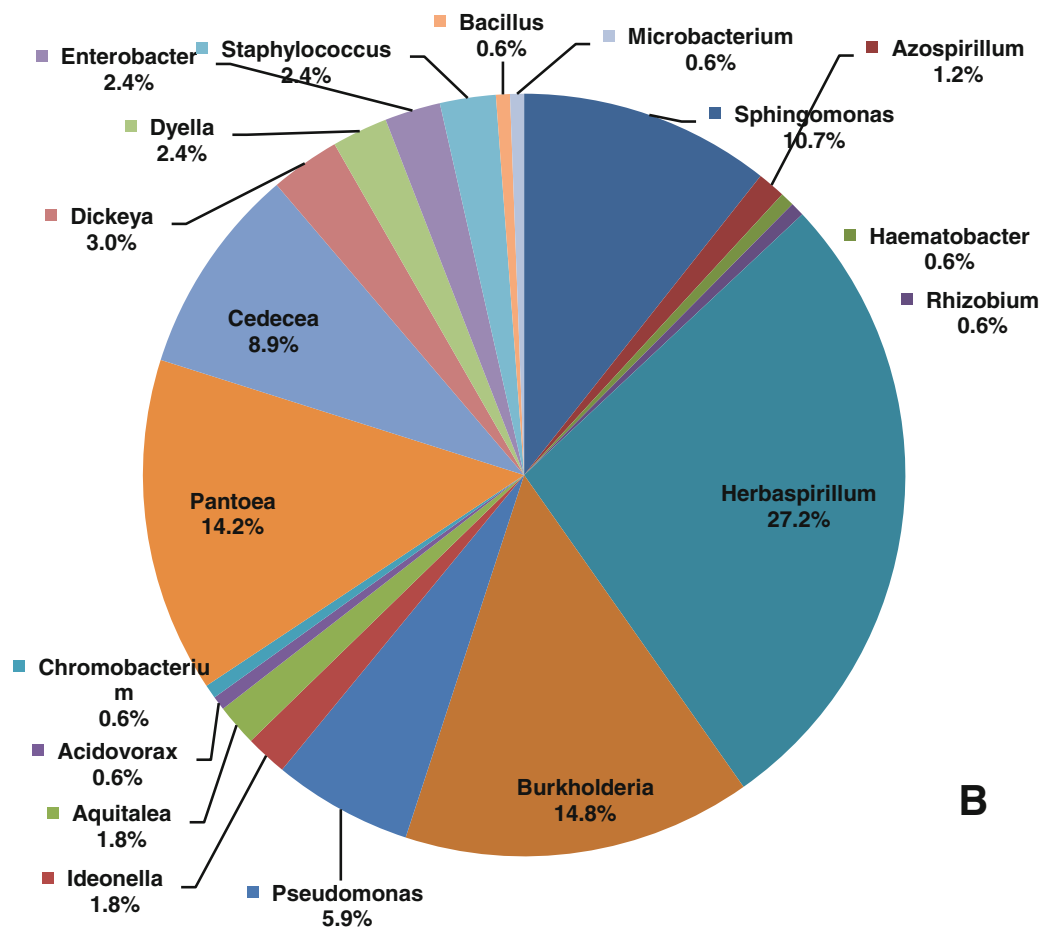

proteobacteria, and the $\beta$-proteobacteria group was predominated. Compared with R-R-WF, R-R-MV rotation witnesses a $5.2 \%$ decreased in $\alpha$-proteobacteria, a $14.1 \%$ increased in $\beta$-proteobacteria, a $1.9 \%$ increased in $\gamma$ proteobacteria, and a $5.3 \%$ decreased in $\delta$-proteobacteria.

Large amount of Firmicutes and Bacteroidetes were distributed in the rice roots. The proportions of Firmicutes in rice roots with R-R-WF and R-R-MV treatments were 9.3 and $4.5 \%$, respectively, and the proportions of Bacteroidetes were 7.9 and $8.7 \%$, respectively. Through long-term R-R-MV rotation, Firmicutes reduced by $4.8 \%$ and Acidobacteria reduced by $1.9 \%$, while Bacteroidetes increased by $0.9 \%$ and Actinobacteria increased by $1.0 \%$. Other bacteria in rice roots of both R-R-WF and R-R-MV were relative few, and 


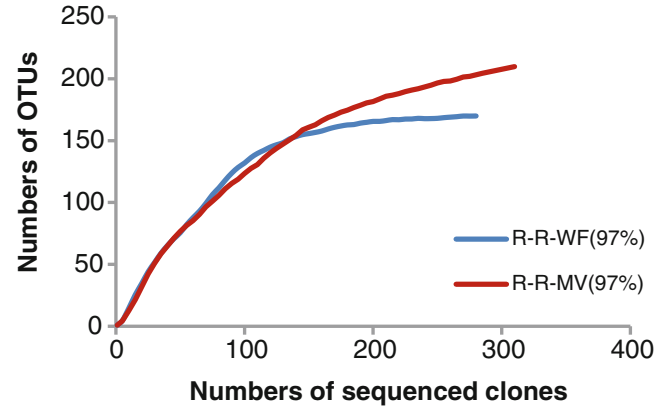

Fig. 3 Rarefaction curve of endophytic bacterial $16 \mathrm{~S}$ rDNA clone library from the rice roots of rice-rice-winter follow $(R-R-W F)$ and rice-riceChinese milk vetch $(R-R-M V)$ rotation. Curves were produced at a cutoff of 0.03 for each sample

their proportions to the total clone number were all below $2 \%$. Armatimonadetes was not found in R-R-MV rotation.

\section{Discussions}

Comparative Analysis of Endophytic Bacteria in Rice Roots Between Culture-Dependent and Culture-Independent Methods

According to the comparison of the compositions at class level between the isolates and colonies from the 16S rDNA clone library, the dominant group in roots of rice after both R-R-WF and R-R-MV rotations were $\beta$-proteobacteria in Proteobacteria, which was consistent with previous reports $[2,13,30]$. In the cultivated method, Proteobacteria account for 96.1 and $96.5 \%$, respectively; in the clone library of the $16 \mathrm{~S}$ rDNA gene, they accounted for 68.9 and $74.5 \%$ of the total clones, respectively. The cultivated method did not isolate the strains of $\delta$ proteobacteria class group but large amount of the clones were found in the culture-independent method (15.7-17.5\%). $\delta$ proteobacteria seldom appeared as endophytic bacteria in reports, and even when they did, they were not regarded as the major community [30]. In our clone library, however, they are taken as the third largest group, proving the richness in diversity of endophytic bacteria from rice roots. Communities such as Bacteroidetes and Acidobacteria were detected by cultureindependent method but did not obtain the pure cultures.

Table 2 Comparison of numbers of OTUs, richness, diversity, and coverage at evolutionary distances of 0.03 according to MOTHUR results

\begin{tabular}{lllllll}
\hline & No. OTUs & Coverage $C$ & Chao 1 & ACE & $H^{\prime}$ & \multicolumn{1}{l}{$D$} \\
\hline R-R-WF & 177 & $22.59 \%$ & 509.71 & $1,671.09$ & 4.84 & 0.01 \\
R-R-MV & 182 & $20.88 \%$ & 629.65 & $1,719.84$ & 4.67 & 0.02 \\
\hline
\end{tabular}

Chao 1 richness estimator, $A C E$ abundance-based coverage estimator, $H^{\prime}$ diversity, Shannon index, $D$ Simpson index
With the cultivated method, only 1 of 246 isolated had a 16S rRNA gene similarity with the known species less $97 \%$ and others over $99 \%$. With the culture-independent method, however, 359 OTUs were obtained from two treatments, and the similarity of $52.9 \%$ OTUs of the known species was below $97 \%$, the similarity of $39.0 \%$ of OTUs of the known species was below $94 \%$. It is thus concluded that a huge number of uncultured bacteria exist in rice roots and a welldesigned culture medium is helpful in isolating novel strains and discovering new functions of microbial colonies.

Strains isolated with the medium R2A and grouped into the genus of Herbaspirillum were the dominant colonies in the two tillage systems, but in the 590 clone sequences obtained with the culture-independent method, only 7 showed high similarity to Herbaspirillum. Therefore, the two methods could be complementary to each other in the study of microbial communities.

Effects of Various Cropping Systems on the Compositions and Diversity of Endophytic Bacteria

Large amounts of endophytic bacteria from the roots, stems, leaves, and seeds of rice were isolated. The reported rice endophytic bacteria were categorized into 64 species of 33 genera, mainly including genera Pantoea, Methylobacterium, Bacillus, Pseudomonas, Burkholderia and Phyllobacterium, Enterobacter, Erwinia, and Klebsiella [20]. Particular communities were always found in certain parts of rice, such as Pantoea in seeds, Methylobacterium in stems, Azpspirillum and Herbaspirillum with the nitrogen function in roots and stems, etc. The culture-dependent study proved that the Herbaspirillum was the common dominant colony in the two treatments. Previous studies had proved the Herbaspirillum as regular endophytic bacteria in plants such as rice [26], sugarcane [23], and corn [21]. Herbaspirillum was also conductive to fix nitrogen and promote plant growth [5], but it was not the dominant endophytic colony. In this experiment, as rice had long been grown in the experiment site, we believe a mutually beneficial relationship may have been established between the Herbaspirillum and rice so that colonies could accumulate, and they are the result of the long-term chronic co-evolution. The large amount of considerable the genus Cedecea was found in rice roots both in R-R-MV and R-R-WF rotations (20.8 and $8.9 \%$, respectively). The genus Cedecea is mostly isolated from human and animal bodies and was believed to be a possible pathogenic bacteria; until recently, two strains of Cedecea davisae were first reported by Silva et al. [28] to have been isolated from plants and were first found to be able to inhibit coffee diseases, but there was no other report except for this one. This study firstly isolated the strains from rice roots as one of the major colonies, so its ecological function needs further research studies. 
Fig. 4 Class level comparison of endophytic bacteria distribution in R-R-WF (a) and R-R-MV (b) rice roots based on cultureindependent method
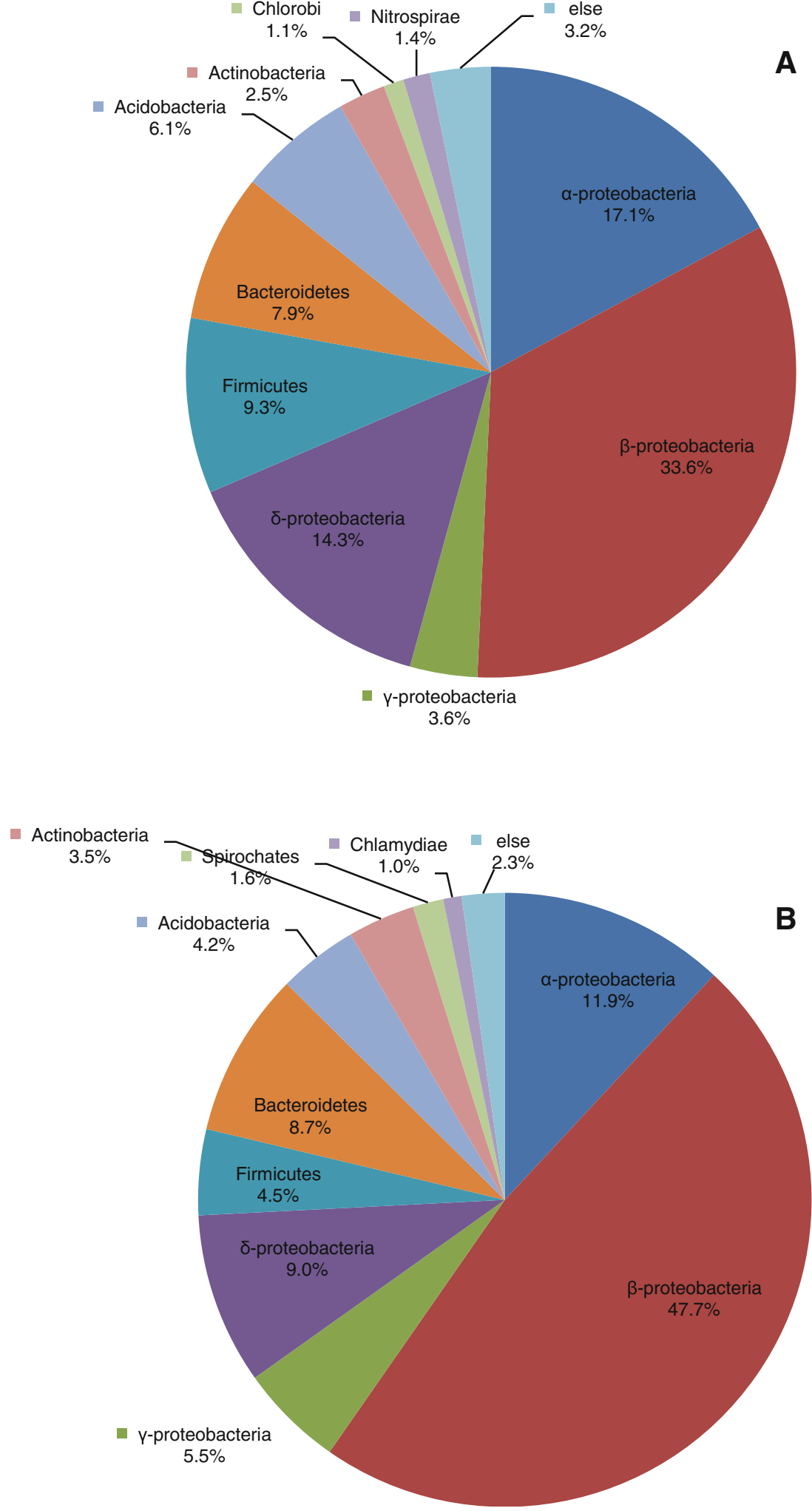

This study reveals much higher proportion of Burkholderia, Pantoea, Sphingomonas, and Pseudomonas isolated from RR-MV rice than that from R-R-WF rice; these genera are common endophytic bacteria in plants and can promote plant growth according to reports [11, 14], whereas according to this study, Haematobacter, Rhizobium, Chromobacterium, Enterobacter, and Microbacterium were specific groups to RR-MV rice; the Pandoraea, a thiosulfate-oxidizing bacteria 
[10], was the only specific group to R-R-WF rotation. The numbers of cultivated bacteria and the diversity indexes such as the Margalef index, Shannon-Wiener index, and Simpson index from R-R-MV rotation were all higher than those of R-RWF rotation, indicating more diverse endophytic bacteria, although there was no research on the effects of rotation on endophytic bacteria, reports on the influence of rotation on soil microbes have already existed. Turco et al. [34] studied the effects of autochthonous bacteria in continuous corn and rotated corn-soybean on the growth of corn, indicating that continuous cropping leaded to increasing numbers of bacteria which suppressed the growth of corn while rotation gave birth to a lower percentage of such bacteria; Peters et al. [24] found that rotation enhanced the antibiosis abilities of endophytic and rhizosphere bacteria (endo- and exoroot) against pathogenic fungi; community level physiological profiles indicated that rotation cropping systems tended to have higher overall microbial activity compared to continuous cropping systems $[18,36]$. According to random amplified polymorphic DNA analysis, rotation cropping of cucumbers showed higher soil microbial activity, microbial biomass, and Shannon-Weaver index than continuous cropping [36]. All the above studies indicate that crop rotation exerted influence upon soil microbial structure and activity, and enabled microbes to play beneficial roles, which was consistent with the results of this research. The 28year experiments on this site have demonstrated that rice yields in R-R-MV rotation system were higher than in R-R-WF system [7], which might be associated with the accumulation of beneficial microbes and more abundant bacterial communities.

Considering the limitations of the culture-dependent method, the culture-independent method was used in this study to further study the effects of different rotation systems on rice endophytic bacteria. The endophytic bacteria in R-R-MV and R-R-WF systems showed similar Shannon-Weaver indexes (4.84 and 4.67), but the Simpson index of the former was obviously more than twice that of the latter, and the Chao 1 index and the ACE index of the former were also significantly higher than those of the latter. Species richness and environmental complexity are proved to increase ecosystem functioning [17]. Meanwhile, endophytes play an important role in ecological community by protection and survival value to the plants, such as enhancement of stress-, insect- and diseaseresistance, and growth promotion [11, 14, 20, 21, 28]. Therefore, we presume that the high richness and diversity of endophytic bacteria in R-R-MV are beneficial to the growth and productivity of rice. The composition of rice endophytic bacteria in different rotation systems also varied. The proportion of $\alpha$-proteobacteria, $\delta$-proteobacteria, Firmicutes, and Acidobacteria in R-R-MV system decreased compared with $\mathrm{R}-\mathrm{R}-\mathrm{WF}$ system and the proportion of $\beta$-proteobacteria increased by $14.1 \%$. These figures indicated that long-term various tillage systems exerted a relatively significant influence on the composition of microbes. Previous reports made indirect assessment of the effects of green manure on soil microbes using microbial biomass and enzyme activity characteristics [29, 32, 35]. This study offered direct verification of the above-mentioned results. Whether the culturable or culture-independent method was used, it was confirmed that the rotation system increased the number of rice endophytic microbes and the diversity level of colonies, in spite of variations in the detected bacteria composition.

\section{Conclusion}

Compared with R-R-WF, R-R-MV consisted of more abundant bacteria communities in rice roots. The diversity level was higher and the composition of colonies was significantly different. Based on this study, further research studies were expected to be made on composition difference in functional microbial communities like nitrogen cycle-related microbes, correlation of microbial composition and rice yield, physical and chemical properties of soil, providing more information for the microbial ecosystem of paddy fields with green manure.

Acknowledgments This work was supported by the National Natural Science Foundation of China (grant nos. 41271273, 41025004), the National High Technology Research and Development Program of China (2012AA101403), and Special Scientific Research Funds for Commonwealth Agricultural Industry (201103005-01).

\section{References}

1. Bending GD, Turner MK, Rayns F, Marx MC, Wood M (2004) Microbial and biochemical soil quality indicators and their potential for differentiating areas under contrasting agricultural management regimes. Soil Biol Biochem 36:1785-1792

2. Chelius MK, Triplett EW (2001) The diversity of archaea and bacteria in association with the roots of Zea mays L. Microb Ecol 41:252263

3. Compant S, Clément C, Sessitsch A (2010) Plant growth-promoting bacteria in the rhizo- and endosphere of plants: their role, colonization, mechanisms involved and prospects for utilization. Soil Biol Biochem 42:669-678

4. Drinkwater LE, Wagoner P, Sarrantonio M (1998) Legume-based cropping systems have reduced carbon and nitrogen losses. Nature 396:262-265

5. Elbeltagy A, Nishioka K, Sato T, Suzuki H, Ye B, Hamada T, Isawa T, Mitsui H, Minamisawa K (2001) Endophytic colonizationand in planta nitrogen fixation by a Herbaspirillum sp. isolated from wild rice species. Appl Environ Microbiol 67(11):5285-5293

6. Gao JS, Cao WD, Li DC, Xu MG, Zeng XB, Nie J, Zhang WJ (2011) Effects of long-term double-rice and green manure rotation on rice yield and soil organic matter in paddy field. Acta Ecologica Sinica 31(16):4542-4548

7. Gao JS, Xu MG, Qin DZ (2008) Effects of long-term rotation of rice, rice and milk vetch on growth and yield in rice. Hunan Agric Sci $6: 25-27$ 
8. Garbeva P, van Overbeek LS, van Vuurde JWL, van Elsas JD (2001) Analysis of endophytic bacterial communities of potato by plating and denaturing gradient gel electrophoresis (DGGE) of 16S rDNA based PCR fragments. Microb Ecol 41:369-383

9. Good IL (1953) The population frequencies of species and the estimation of population parameters. Biometrika 40:237-264

10. Graff A, Stubner S (2003) Isolation and molecular characterization of thiosulfate-oxidizing bacteria from an Italian rice field soil. Syst Appl Microbiol 26(3):445-452

11. Hardoim PR, van Overbeek LS, van Elsas JD (2008) Properties of bacterial endophytes and their proposed role in plant growth. Trends Microbiol 16(10):463-471

12. Hill TCJ, Walsh KA, Harris JA, Moffett BF (2003) Using ecological diversity measures with bacterial communities. FEMS Microbiol Ecol 43:1-11

13. Kaiser O, Pühler A, Selbitschka W (2001) Phylogenetic analysis of microbial diversity in the rhizoplane of oilseed rape (Brassica napus cv. Westar) employing cultivation-dependent and cultivationindependent approaches. Microb Ecol 42:136-149

14. Kang SH, Cho HS, Cheong H, Ryu CM, Kim JF, Park SH (2007) Two bacterial entophytes eliciting both plant growth promotion and plant defense on pepper (Capsicum annuum L.). J Microbiol Biotechnol 17(1):96-103

15. Kim OS, Cho YJ, Lee K, Yoon SH, Kim M, Na H, Park SC, Jeon YS, Lee JH, Yi H, Won S, Chun J (2012) Introducing EzTaxon-e: a prokaryotic 16S rRNA gene sequence database with phylotypes that represent uncultured species. Int J Syst Evol Microbiol 62:716-721

16. Lane DJ (1991) 16S/23S rRNA sequencing. In: Stackebrandt E, Goodfellow M (eds) Nucleic acid techniques in bacterial systematics. Wiley, New York, pp 115-175

17. Langenheder S, Bulling MT, Solan M, Prosser JI (2010) Bacterial biodiversity-ecosystem functioning relations are modified by environmental complexity. PLoS ONE 5(5):e10834. doi:10.1371/journal. pone. 0010834

18. Larkin RP (2003) Characterization of soil microbial communities under different potato cropping systems by microbial population dynamics, substrate utilization, and fatty acid profiles. Soil Biol Biochem 35:1451-1466

19. MacRae RJ, Mehuys GR (1985) The effect of green manuring on the physical properties of temperate-area soils. Adv Soil Sci 3:71-94

20. Mano H, Morisaki H (2008) Endophytic bacteria in the rice plant. Microb Environ 23:109-117

21. Montañez A, Blanco AR, Barlocco C, Beracochea M, Sicardi M (2012) Characterization of cultivable putative endophytic plant growth promoting bacteria associated with maize cultivars (Zea mays L.) and their inoculation effects in vitro. Appl Soil Ecol 58:21-28

22. Morrissey JP, Dow JM, Mark GL, O'Gara F (2004) Are microbes at the root of a solution to world food production? EMBO Rep 5(10): 922-926
23. Pariona-Llanos R, Ferrara FIS, Soto HH, Barbosa HR (2010) Influence of organic fertilization on the number of culturable diazotrophic endophytic bacteria isolated from sugarcane. Eur J Soil 46(6):387-393

24. Peters RD, Sturz AV, Carter MR, Sanderson JB (2003) Developing disease-suppressive soils through crop rotation and tillage management practices. Soil Till Res 72:181-192

25. Robertson GP, Paul EA, Harwood RR (2000) Greenhouse gases in intensive agriculture: contributions of individual gases to the radioactive forcing of the atmosphere. Science 289:1922-1925

26. Roncato-Maccari LDB, Ramos HJO, Pedrosa FO, Alquini Y, Chubatsu LS, Yates MG, Rigo LU, Steffens MBR, Souza EM (2003) Endophytic Herbaspirillum seropedicae expresses nif genes in gramineous plants. FEMS Microbiol Ecol 45(1):39-47

27. Schloss PD, Westcott SL, Ryabin T, Hall JR, Hartmann M, Hollister EB, Lesniewski RA, Oakley BB, Parks DH, Robinson CJ, Sahl JW, Stres B, Thallinger GG, Van Horn DJ, Weber CF (2009) Introducing mothur: open-source, platform-independent, community supported software for describing and comparing microbial communities. Syst Appl Microbiol 75:7537-7541

28. Silva HSA, Tozzi JPL, Terrasan CRF, Bettiol W (2012) Endophytic microorganisms from coffee tissues as plant growth promoters and biocontrol agents of coffee leaf rust. Biol Control 63(1):62-67

29. Stark C, Condron LM, Stewart A, Di HJ, O'Callaghan M (2007) Influence of organic and mineral amendments on microbial soil properties and processes. Appl Soil Ecol 35:79-93

30. Sun L, Qiu FB, Zhang XX, Dai X, Dong XZ, Song W (2008) Endophytic bacterial diversity in rice (Oryza sativa L.) roots estimated by $16 \mathrm{~S}$ rDNA sequence analysis. Microb Ecol 55:415-424

31. Tamura K, Peterson D, Peterson N, Stecher G, Nei M, Kumar S (2011) MEGA5: molecular evolutionary genetics analysis using maximum likelihood, evolutionary distance, and maximum parsimony methods. Mol Biol Evol 28:2731-2739

32. Tejada M, Gonzalez JL, García-Martínez AM, Parrado J (2008) Effects of different green manures on soil biological properties and maize yield. Bioresource Technol 99:1758-1767

33. Thompson JD, Gibson TJ, Plewniak F, Jeanmougin F, Higgins DG (1997) The CLUSTAL_X windows interface: flexible strategies for multiple sequence alignment aided by quality analysis tools. Nucleic Acids Res 25:4876-4882

34. Turco RF, Bischoff M, Breakwell DP, Griffith DR (1990) Contribution of soil-borne bacteria to the rotation effect in corn. Plant Soil 122: 115-120

35. Yang ZP, Gao JS, Zheng SX, Nie J, Xu MG, Xie J, Liao YL (2011) Effects of long-term winter planting-green manure on microbial properties and enzyme activities in reddish paddy soil. Soils 43(4): 576-583

36. Yao HY, Jiao XD, Wu FZ (2006) Effects of continuous cucumber cropping and alternative rotations under protected cultivation on soil microbial community diversity. Plant Soil 284:195-203 